\title{
Light quarks on the lattice: methods and results for pion physics
}

\section{Stefan Schaefer ${ }^{* \dagger}$}

Humboldt Universität zu Berlin, Institut für Physik, Newtonstr. 15, 12489 Berlin, Germany

E-mail: stefan.schaefer@cern.ch

\begin{abstract}
Ab-initio studies of the physics of pions using lattice QCD have become possible over the last decade, where particular attention was given to the computation of the constants of the chiral Lagrangian. This is due to significant progress in algorithms, which now allow simulations with light sea quarks on fine lattices.

This short writeup has two objectives: the first is to introduce the ideas behind the algorithmic advances along with the status and prospects of the simulations. After that, the state of the physics results will be reviewed, with particular emphasis on the chiral low-energy constants.
\end{abstract}

35th International Conference of High Energy Physics - ICHEP2010,

July 22-28, 2010

Paris France

\footnotetext{
* Speaker.

†Present address: CERN, Physics department, 1211 Geneva 23, Switzerland
} 


\section{Introduction}

In recent years, a significant part of the efforts in lattice QCD were directed towards problems involving light quarks. New algorithms allow calculations with sea quarks at masses down to the physical values. This makes it possible to learn more about the physics involving these quarks. In particular, the physics of chiral symmetry and its spontaneous breaking. These two topics will be covered in this very short write-up.

\section{Light quarks: algorithms}

Why was the simulation of light quarks so expensive 10 years ago? Basically, because algorithms were used, which did not encode the relevant physics. The fermion determinant was introduced in a single, stochastic estimate, introducing a lot of artificial noise into the calculation. They also did not take into account that the fermion physics of the infra-red is different from the ultra-violet. Progress has therefore been made along the line of better estimates of the fermion action and separation of the ultra-violet from the infra-red part. The two parts are dominated by significantly different frequencies: the equations of motion, which govern the evolution of the gauge fields during the simulation, can be integrated on much longer time scales for the IR part of the action than for the UV part.

The initial break-through in both directions comes from Hasenbusch's mass preconditioning[1]. Starting from the seemingly simple identity $\operatorname{det} D(m)=\operatorname{det}\left[D(m) D^{-1}(M)\right] \operatorname{det} D(M)$, with $D(m)$ the Dirac operator of mass $m$ and $M>m$, both can be achieved: the combined stochastic estimate of the two factors is less noisy than the estimate of the left hand side alone. And also the $D(M)$, the Dirac operator for a heavy quark, is dominated by the UV, whereas the rest in the first term on the right hand side is largely IR. Similarly successful algorithms are based on a domain decomposition, a geometric UV/IR separation via blocks of a size smaller than the IR scales of QCD[2]. Also the RHMC[3] based on the identity $\operatorname{det} D=\prod_{n} \operatorname{det} \sqrt[n]{D}$ is widely used these days, in particular for non-degenerate fermions like the strange and charm.

The second area of improvements is in the methods to solve the Dirac equation $D(m) \psi=\phi$. Whereas ten years ago, a scaling of the cost with the inverse quark mass seemed unavoidable, costefficient preconditioning methods which incorporate the infra-red physics of the fermions have almost eliminated this dependence[4].

A third area of improvement was in the way to compute observables. Stochastic estimates have essentially replaced the old point sources, averaging over all source positions[5]. In small volume, computing the IR contribution exactly has proven very efficient as well[6].

For any reliable computation, it is an absolute necessity to extrapolate the result of several fine lattices to the continuum. However, it turns out that in the range of current lattice simulations, the cost of doing so rises with roughly the tenth power of the inverse lattice spacing, because observables like the topological charge show a very severe critical slowing down[7]. This challenge remains to be solved during the next years for trustworthy computations.

\section{Light quarks: Chiral low energy constants}

The improvements mentioned in the previous section have made it possible for many collabo- 

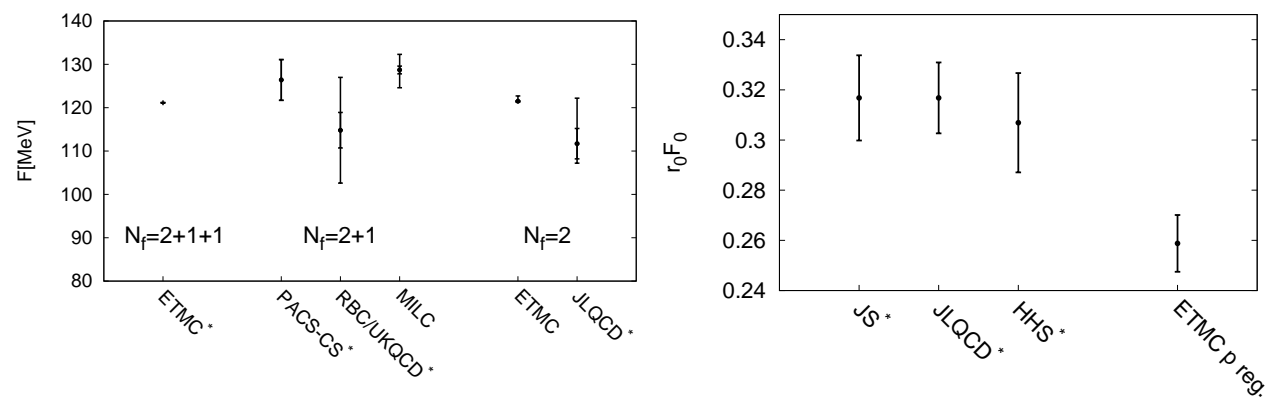

Figure 1: Results for the decay constant $F$, the $p$-regime values are given on the left plot, the $\varepsilon$-regime results on the right in units of the scale $r_{0} \approx 0.5 \mathrm{fm}[12]$, along with the $p$-result from ETMC for comparison. The star indicates that the continuum limit is not taken. $\varepsilon$-regime results are $N_{f}=2$.

rations to generate data at light pion masses, which are within the applicability of chiral perturbation theory (ChPT) and whose assumptions can therefore be tested. This is done by matching the predictions of $\mathrm{ChPT}$ to the lattice data, which in turn also fixes the chiral low energy constants of the ChPT Lagrangian. Lattice computations now render some of the most precise determinations of these constants.

ChPT is an expansion around zero quark mass and only expected to give a reasonable description within a certain mass range. At the cost of more low energy constants (LEC), higher orders will give higher accuracies, but the range of applicability will not necessarily be much larger. Lighter quark masses lead to systematic improvement tough. Most collaborations set a limit to the applicability of ChPT around pion masses $500 \mathrm{MeV}$, though this is under debate.

A further problem arises from the fact that computations are done at finite lattice spacing $a$. The unambiguous procedure would be to first extrapolate the observables to the continuum and then perform the matching. This is complicated and therefore most groups either match the continuum ChPT to finite $a$ data and then extrapolate the LECs to $a=0$. Others use a ChPT which includes finite $a$ effects[8] but comes with additional constants whose values depend on the particular discretized action used in the numerical lattice computation. Both approaches are problematic from either the conceptual or practical point of view.

For a comprehensive discussion of the extraction of LECs on the lattice, clearly beyond the scope of this text, see the Flavianet report[9]. Only results of the two leading order LECs are shown here. The results for the decay constant $F$ from p-regime[10] and $\varepsilon$-regime[11] calculations can be found in Fig. 1. The $p$-regime is a large volume regime, with small corrections due to the finite size, whereas the $\varepsilon$-regime provides a finite size scaling setup. The two complementary approaches are used to get a handle on the finite volume systematics. The overall agreement is rather good despite the fact that the flavor content of the sea differs and not all results are in the continuum.

For the chiral condensate $\Sigma$ in Fig. 2, however, there is some tension in the results, probably due to the more problematic renormalization. The renormalization procedures employed by the different groups have varying systematics, frequently relying on perturbation theory. Further differences arise from the quantities used to set the lattice spacing $a$, which is ambiguous if not the full standard model physics is considered. 

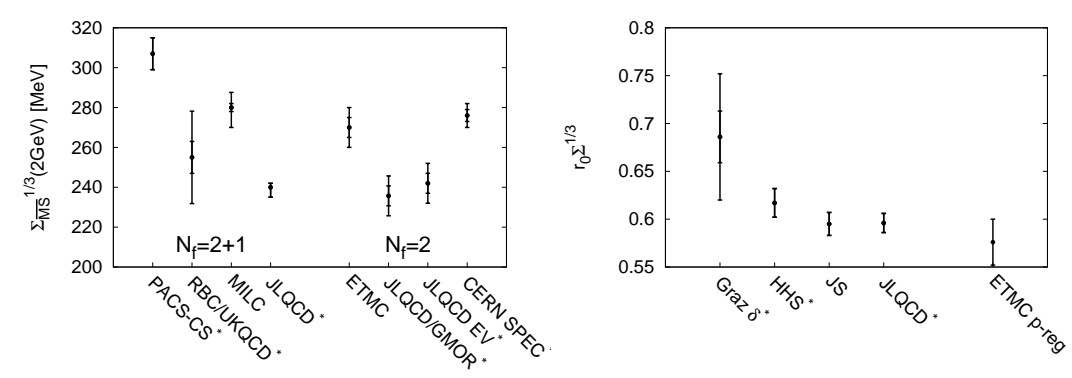

Figure 2: Same as Fig. 1, but for the chiral condensate $\Sigma$.

\section{Summary}

The progress in lattice QCD has allowed to get a much better control over the systematic errors of observables related to the physics of pions than only a few years ago. The next years will bring more results at physical light quark masses and realistic flavor content in the sea. The continuum limit will remain a challenge, but also at the current lattice spacings around $0.05 \mathrm{fm}$, interesting results can be achieved.

\section{References}

[1] M. Hasenbusch Phys. Lett. B519 (2001) 177-182.

[2] M. Lüscher Comput. Phys. Commun. 165 (2005) 199;

[3] M. Clark and A. Kennedy Phys.Rev.Lett. 98 (2007) 051601.

[4] M. Lüscher JHEP 07 (2007) 081.

[5] R. Sommer Nucl.Phys.Proc.Suppl. 42 (1995) 186-193.

[6] T. A. DeGrand and S. Schaefer, Comput.Phys.Commun. 159 (2004) 185-191;

L. Giusti, P. Hernandez, M. Laine, P. Weisz, and H. Wittig, JHEP 0404 (2004) 013.

[7] S. Schaefer, R. Sommer, and F. Virotta, arXiv:1009.5228.

[8] S. R. Sharpe, R. L. Singleton, Jr, Phys. Rev. D58 (1998) 074501.

[9] G. Colangelo, S. Durr, A. Juttner, L. Lellouch, H. Leutwyler, et. al. arXiv: 1011.4408.

[10] PACS-CS Collaboration, S. Aoki, et. al. Phys.Rev. D79 (2009) 034503;

RBC-UKQCD Collaboration, C. Allton, et. al. Phys.Rev. D78 (2008) 114509;

MILC Collaboration, A. Bazavov et. al., PoS CD09 (2009) 007.

ETM Collaboration R. Baron et al., JHEP 1008, 097 (2010).

ETM Collaboration, R. Baron, et al., JHEP 1006 (2010) 111.

L. Giusti, M. Lüscher, JHEP 0903 (2009) 013.

JLQCD and TWQCD Collaboration, J. Noaki et al., Phys. Rev. Lett. 101 (2008) 202004.

[11] A. Hasenfratz, R. Hoffmann, S. Schaefer, Phys. Rev. D78 (2008) 054511.

O. Bar, S. Necco, S. Schaefer, JHEP 0903 (2009) 006.

K. Jansen, A. Shindler, PoS LAT2009 (2009) 070.

C. B. Lang, P. Majumdar, W. Ortner, Phys. Lett. B649 (2007) 225-229.

H. Fukaya et al. [ JLQCD Collaboration ], Phys. Rev. D77 (2008) 074503.

[12] R. Sommer, Nucl. Phys. B411 (1994) 839-854. 